Синдром Швахмана - Даймонда: современные генетические аспекты заболевания из группы рибосомопатий

\author{
М.Г. Ипатова ${ }^{1,2}$ \\ ${ }_{1}^{1}$ ФГАОУ ВО «Российский национальный исследовательский медицинский университет имени Н.И. Пирогова» Министерства \\ здравоохранения Российской Федерации; Россия, г. Москва \\ 2 ГБуз «Городская детская клиническая больница имени Н.Ф. Филатова Департамента здравоохранения города Москвы»; Россия, \\ 2. Москва
}

\title{
PЕЗЮМЕ
}

\begin{abstract}
Цель обзора: проанализировать новые мутации в генах DNAJC21, EFL1, SRP54, приводящие к нарушению биогенеза рибосом и проявляющиеся клиническими симптомами, схожими с синдромом Швахмана - Даймонда (СШД).

Основные положения. СШД относится к заболеваниям из группы рибосомопатий и характеризуется экзокринной недостаточностью поджелудочной железы, нарушением гемопоэза, скелетными аномалиями и высоким риском развития миелодиспластического синдрома и острого миелоидного лейкоза. Около 90\% пациентов с СШД имеют биаллельные мутации в гене SBDS. Однако у 10-20\% пациентов с симптомокомплексом, характерным для СШД, патогенные варианты в гене SBDS не идентифицируются, что послужило причиной поиска других генов-кандидатов. К наследственным заболеваниям, связанным с дефектами созревания, нарушением структуры или функции рибосом и рибонуклеопротеиновых комплексов, помимо СШД, относят анемию Даймонда - Блекфена, гипоплазию хрящей и волос (метафизарную хондродисплазию, тип Мак-Кьюсика), врожденный дискератоз, 5q-синдром и другие. Эти синдромы имеют общие характеристики с СШД. Все заболевания связаны с костномозговой недостаточностью по меньшей мере одной линии гемопоэза. Все пять перечисленных синдромов ассоциируются с повышенным риском развития рака.

Заключение. СШД - это генетически детерминированное заболевание из группы рибосомопатий. Рибосомопатии обусловлены мутациями в генах, участвующих в синтезе рибосомальных белков и факторов, которые функционируют на различных стадиях их сборки, вызывают широкий спектр клинических фенотипов, включая злокачественные гематологические заболевания и рак. В клинической практике традиционно диагноз СШД устанавливают по характерным клиническим симптомам и при наличии патогенных мутаций в гене SBDS. Относится ли СШД к генетически гетерогенным заболеваниям из группы рибосомопатий, или мутации в других генах, приводящих к нарушению биогенеза рибосом, вызывают СШД-подобные симптомы, остается вопросом дискутабельным и требует дальнейших исследований.

Ключевые слова: синдром Швахмана - Даймонда, гены, SBDS, DNAJC21, EFL1, SRP54, рибосомопатия.
\end{abstract}

Конфликт интересов: автор заявляет об отсутствии конфликта интересов.

Для цитирования: Ипатова М.Г. Синдром Швахмана - Даймонда: современные генетические аспекты заболевания из группы рибосомопатий. Доктор.Ру. 2020; 19(10): 33-36. DOI: 10.31550/1727-2378-2020-19-10-33-36

\section{Shwachman - Diamond Syndrome: Modern Genetic Aspects of a Ribosomapathy}

\author{
M.G. Ipatova ${ }^{1,2}$ \\ ${ }^{1}$ N.I. Pirogov Russian National Research Medical University (a Federal Government Autonomous Educational Institution of Higher \\ Education), Russian Federation Ministry of Health; 1 Ostrovityanov St., Moscow, Russian Federation 117997 \\ ${ }^{2}$ N.F. Filatov Municipal Clinical Children Hospital of Moscow Department of Health; 15 Sadovaya-Kudrinskaya Str., Moscow, Russian \\ Federation 123001
}

\section{ABSTRACT}

Objective of the Review: To analyse new DNAJC21, EFL1, SRP54 mutations causing ribosome biogenesis defects and presenting with clinical symptoms similar to the symptoms of Shwachman - Diamond syndrome (SDS).

Key Points. SDS is a ribosomapathy and is characterised by pancreatic exocrine insufficiency, defective hematopoiesis, musculoskeletal anomalies, and a high risk of myelodysplastic syndrome and acute myeloid leukemia. About $90 \%$ of SDS patients have biallelic SBDS mutations. However, $10-20 \%$ of patients with a set of symptoms typical of SDS did not have any pathovars in SBDS gene; therefore, we searched for other candidate genes. In addition to SDS, genetic disorders associated with defected maturation, deficient structure or function of ribosomes and ribonucleoprotein complexes include Diamond - Blackfan anemia, cartilage and hair hypoplasy (McKusick type metaphyseal chondrodysplasia), congenital diskeratosis, 5q-syndrome, and others. These syndromes are similar to SDS. All these conditions are associated with medullary deficiency at least in one hematopoiesis chain. All five conditions are associated with a high risk of cancer.

Conclusion. SDS is a genetically determined condition belonging to ribosomapathies. Ribosomapathies are caused by mutations in genes that participate in the synthesis of ribosomal proteins and factors, functioning at various stages of their assembly, and give origin to a number of clinical phenotypes, including haematological malignancies and cancer. In clinical practice, SDS is diagnosed on the basis of typical clinical

Ипатова Мария Георгиевна - к. м. н., руководитель гепатобилиарного центра ГБУз «ДГКБ № 13 им. Н.Ф. Филатова ДЗМ», доцент кафедры госпитальной педиатрии имени академика В.А. Таболина педиатрического факультета ФГАОУ ВО «РНИМУ им. Н.И. Пирогова» Минздрава России. 117997, Россия, г. Москва, ул. Островитянова, д. 1. еLIBRARY.RU SPIN:1837-5380. http://orcid.org/0000-0003-0295-4820. E-mail: mariachka1@mail.ru 
symptoms and if pathogenic SBDS mutations are present. The issue whether SDS is a genetic heterogenetic ribosomapathy or a mutation of other genes causing defective ribosome synthesis and SDS-like symptoms, is disputable and requires further research.

Keywords: Shwachman - Diamond syndrome, genes, SBDS, DNAJC21, EFL1, SRP54, ribosomapathy.

Conflict of interest: The authors declares that she does not have any conflict of interests.

For citation: Ipatova M.G. Shwachman - Diamond Syndrome: Modern Genetic Aspects of a Ribosomapathy. Doctor.Ru. 2020; 19(10): 33-36. (in Russian). DOI: 10.31550/1727-2378-2020-19-10-33-36

C: индром Швахмана - Даймонда (СШД; Shwachman Diamond syndrome, OMIM 260400) - редкое мультисистемное заболевание, характеризующееся преимущественно нарушением гемопоэза, экзокринной недостаточностью поджелудочной железы, задержкой физического развития и аномалиями костной системы [1-3]. Помимо вышеописанных симптомов, при СШД имеются иммунологические нарушения, поражение печени в виде повышения активности трансаминаз, повреждение эмали зубов, нейрокогнитивные расстройства и другие симптомы $[4,5]$. СШД ассоциируется с высоким риском развития миелодиспластического синдрома (МДС) и злокачественных трансформаций, особенно в острый миелоидный лейкоз (ОМЛ) $[6,7]$.

Болезнь названа в честь врачей Гарри Швахмана (Harry Shwachman) и Луиса Даймонда (Louis Diamond), которые в 1964 г. описали сочетанную патологию недостаточности экзокринной функции поджелудочной железы с гипоплазией костного мозга у трех детей из одной семьи [1].

Распространенность СШД составляет 1 на 76 тыс. населения [8]. Заболеваемость СШД в итальянской популяции составила 1 на 168 тыс. новорожденных [9].

В регистре National Cancer Institute Inherited Bone Marrow Failure Syndromes среди 530 пациентов с недостаточностью костного мозга (анемией Фанкони, врожденным дискератозом, анемией Даймонда - Блекфена и (ШД) лишь у 35 $(6,6 \%)$ человек зарегистрирован СШД [10]. В Канадском регистре из 125 пациентов с недостаточностью кроветворения с СШД наблюдались 34 (27,2\%) [11].

Распространенность СШД в России неизвестна. При анализе эпидемиологических показателей из 2728 больных, зарегистрированных в базе первичных иммунодефицитных состояний, в регистр включены 38 детей с СШД [12].

Около 90\% пациентов с клиническими симптомами СШД имеют биаллельные патогенные варианты в высоко консервативном гене SBDS, который локализован на длинном плече 7 хромосомы (7q11) [2]. Этот ген состоит из 5 экзонов, кодирующих белок SBDS из 250 аминокислотных остатков [2]. Соседний псевдоген SBDSP на 97\% гомологичен с геном $S B D S$, но содержит делеции и изменения нуклеотидов, которые препятствуют образованию функционального белка. Приблизительно у 75\% больных СШД выявлены патогенные варианты, возникающие в результате конверсии гена с псевдогеном [2].

Наиболее частыми мутациями в гене SBDS являются с.258+2T>C и с.183_184delTAinsCT [13-15]. Мутация 258+2Т >C нарушает донорский сайт сплайсинга в интроне 2, В то время как изменение динуклеотида 183-184ТА $\rightarrow$ СТ приводит к стоп-кодону в рамке считывания (K62X) и, как следствие, укорочению белка SBDS. На сегодняшний день не идентифицирован ни один человек, гомозиготный по динуклеотидному варианту (183-184TA $\rightarrow$ CT), так как эта патогенная мутация ведет к полному отсутствию функционального белка SBDS и несовместима с жизнью [2]. Эти данные согласуются с результатами исследований на мышах, у которых аблация белка SBDS была для эмбрионов смертельной [16].
Помимо патогенных вариантов с.258+2T>C и c.183_184delTAinsCT, описаны различные делеции, инсерции, миссенс-, нонсенс-варианты и точечные мутации в гене SBDS $[14,17,18]$.

У $10-20 \%$ пациентов с СШД не идентифицируются мутации в гене $S B D S[2,4,11,19]$, что послужило причиной поиска других генов-кандидатов.

В настоящее время описаны патогенные варианты в генах DNAJC21 или EFL1 у пациентов с клиническим фенотипом СШД [19-21]. У 4 больных с костномозговой недостаточностью, задержкой внутриутробного роста и/или низким ростом выявлены биаллельные мутации в гене DNAJC21. У одного из этих пациентов в возрасте 12 лет развился ОМЛ (мегакариоцитарный подтип, ОМЛ-М7) [20]. В другом исследовании у 4 человек из трех неродственных семей, которым был поставлен клинический диагноз СШД, также обнаружены биаллельные мутации в гене DNAJC21 [21].

В исследовании P. Stepensky и соавт. (2017) у 6 пациентов с панцитопенией, экзокринной недостаточностью поджелудочной железы и скелетными аномалиями выявили мутации в гене EFL1: 4 пациента были гомозиготными по варианту p.R10950 и 2 пациента - гомозиготными по варианту р.M882K в EFL1 [22].

B 2017 г. R. Carapito и соавт. описали вариант миссенс-мутации de novo в гене SRP54 у 3 различных пациентов с врожденной нейтропенией и другими клиническими проявлениями, характерными для СШД. У этих больных уровень матричной PHK SRP54 в костном мозге был в 3,6 раза ниже, чем у здоровых людей. У них также отмечались снижение количества нейтрофилов, нарушение хемотаксиса и уменьшение размеров экзокринной части поджелудочной железы. У 2 пациентов содержание эластазы кала было менее 15 мкг/г при норме более 200 мкг/г [23].

В исследовании, проведенном С. Bellanne-Chantelot и соавт. (2018), патогенные варианты в гене SRP54 выявлены у 66 пробандов из французского регистра пациентов с врожденными нейтропениями. В общей сложности идентифицированы 23 мутации (16 спорадических, 7 семейных) с 7 различными патогенными вариантами в гене SRP54. Почти у всех пациентов нейтропения была с рождения менее $0,5 \times 10^{9}$ г/л, носила персистирующий характер, сочеталась с нарушением созревания промиелоцитов и требовала длительной терапии высокими дозами гранулоцитарного колониестимулирующего фактора.

Нейтропения иногда ассоциировалась с патологией нервной системы $(n=7)$, низким ростом $(n=3)$ и/или экзокринной недостаточностью поджелудочной железы, при которой требовалась ферментозаместительная терапия $(\mathrm{n}=3)$. На основании полученных данных авторы сделали вывод, что патогенные варианты в гене SRP54 вызывает не СШД, а СШД-подобный синдром [24].

Все вышеописанные мутации ведут к нарушению биогенеза и/или функций рибосом (рис.). Биогенез рибосом включает скоординированную экспрессию и сборку 80 рибосомных белков, 4 рибосомных РНК (рРНК) и 76 малых ядрышковых молекул РНК с помощью 200 факторов сборки 


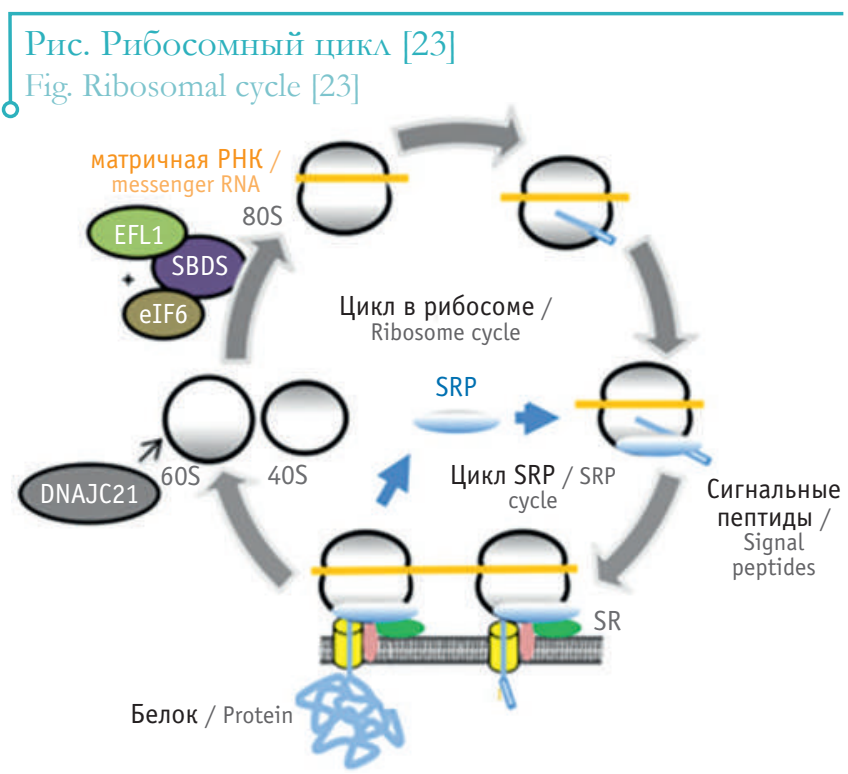

и 300 snoPHК (small nucleolar ribonucleoproteins), выполняющих специфические ковалентные модификации (метилирование, ацетилирование и псевдоуридилирование) [25, 26]. Синтез рибосом начинается с транскрипции двух прерибосомальных РНК: один синтез для $5 \mathrm{~S}$ рРНК, второй - для 18S, $5.8 \mathrm{~S}$ и $28 \mathrm{~S}$ рРНК. В цитоплазме синтезируются все рибосомные белки и транс-активирующие факторы, которые поступают в ядрышко и принимают участие в биогенезе рибосом. Сборка рибосом заканчивается после выхода прерибосомных частиц из ядрышка в цитоплазму [27].

Структура рибосомы включает две субъединицы - субъединицу 40S, состоящую из цепи $18 \mathrm{~S}$ рPHК и 33 видов рибосомальных белков (small ribosomal subunits, RPS), и субъединицу $60 \mathrm{~S}$, состоящую из цепи $28 \mathrm{~S}, 5 \mathrm{~S}$ и $5.8 \mathrm{~S}$ рPHК и 47 видов рибосомальных белков (large ribosomal subunit, RPL) [27]. Вместе 40S и 60S образуют зрелую рибосому $80 \mathrm{~S}$.

Известно, что белок SBDS играет важную роль в биогенезе рибосом и стабильности митотического веретена [28]. В исследовании A.J. Finch и соавт. (2011) показано, что SBDS функционирует на поздней стадии цитоплазматического созревания субъединиц 60S рибосом [29]. Трансдействующим фактором, который удерживает зарождающуюся субъединицу $60 \mathrm{~S}$ в функционально неактивном состоянии, является эукариотический фактор инициации 6 (eIF6). Белок еIF6 связывается с интерсубъединичным мостиком [30] и должен быть удален до того, как субъединица 60S сможет присоединиться к субъединице $40 \mathrm{~S}$ для инициирования трансляции.

В исследовании A.J. Finch и соавт. также показано, что SBDS способствует высвобождению eIF6, стимулируя GTPaseактивность EFL1 [29]. Локализация белка SBDS зависит от клеточного цикла, с преимущественной локализацией в ядрышке во время митоза в фазы G1 и G2 и в ядре во время S-фазы [31].

Белки DNAJC21 и EFL1 тоже участвуют в созревании 60S до начала сборки трансляции 80S рибосомы (см. puc.) $[32,33]$.

Таким образом, мутации в генах SBDS, DNAJC21 и EFL1 ведут к нарушению созревания $60 \mathrm{~S}$ субъединицы, что

\section{ЛИТЕPATYPA / REFERENCES}

1. Shwachman H., Diamond L.K., Oski F.A. et al. The syndrome of pancreatic insufficiency and bone marrow dysfunction. J. Pediatr. 1964; 65: 645-63. DOI: 10.1016/s0022-3476(64)80150-5

2. Boocock G.R., Morrison J.A., Popovic M. et al. Mutations in SBDS are associated with Shwachman-Diamond syndrome. Nat. Genet. 2003; 33(1): 97-101. DOI 10.1016/j.hoc.2009.01.007 вызывает схожие клинические проявления у различных пациентов.

Во время биогенеза и инициации трансляции белка рибосома взаимодействует со многими молекулами-партнерами, и из них наиболее важной является частица распознавания сигналов (signal recognition particle, SRP) (см. puc.). SRP это рибонуклеопротеидный комплекс, способствующий ориентации образующихся полипептидов, несущих сигнальную последовательность на поверхность эндоплазматического ретикулума [34]. SRP млекопитающих состоит из одной молекулы PHК (длиной 300 нуклеотидов 7SL PHK) и 6 полипептидов: SRP9, SRP14, SRP19, SRP54, SRP68 и SRP72 [35].

Как было изложено выше, SRP54 играет центральную роль в рибонуклеопротеидном комплексе, мутации в SRP54 ассоциируются с врожденной нейтропенией и СШД-подобными симптомами [23].

Благодаря своей роли в регулировании качества и количества белков в клетке, биогенез рибосом является неотъемлемой частью клеточного роста, пролиферации и дифференцировки. Нарушение транскрипции рибосомных белков приводит к остановке клеточного цикла, преждевременному старению, апоптозу или аутофагии в зависимости от типа клеток и, как следствие, к нарушению кроветворения и высокому риску онкологических заболеваний [36-38].

На сегодняшний день к наследственным заболеваниям, связанным с дефектами созревания, нарушением структуры или функции рибосом и рибонуклеопротеиновых комплексов, помимо СШД, относят анемию Даймонда - Блекфена (ОМIM 105650), гипоплазию хрящей и волос (метафизарную хондродисплазию, тип Мак-Кьюсика; ОМIM 250250), врожденный дискератоз (ОМІМ 127550 и 305000), 5q-синдром (синонимы: МДС с изолированной $\operatorname{del}(5 q)$, МДC) и другие синдромы [39-44].

Эти синдромы имеют общие характеристики с СШД. Все заболевания связаны с костномозговой недостаточностью по меньшей мере одной линии гемопоэза. Все пять перечисленных синдромов ассоциируются с повышенным риском развития рака. Синдром гипоплазии хрящей и волос, анемия Даймонда - Блекфена и СШД имеют схожие скелетные фенотипы, которые различаются как по тяжести проявлений, так и по пенетрантности [3, 40, 45-47].

\section{ЗАКЛЮЧЕНИЕ}

Синдром Швахмана - Даймонда (СШД) - это генетически детерминированное заболевание из группы рибосомопатий. Рибосомопатии обусловлены мутациями в генах, участвующих в синтезе рибосомальных белков и факторов, которые функционируют на различных стадиях их сборки, вызывают широкий спектр клинических фенотипов, включая злокачественные гематологические заболевания и рак.

В клинической практике традиционно диагноз СШД устанавливают по характерным клиническим симптомам и при наличии патогенных мутаций в гене SBDS. Относится ли СШД к генетически гетерогенным заболеваниям из группы рибосомопатий, или мутации в других генах, приводящих к нарушению биогенеза рибосом, вызывают СШД-подобные симптомы, остается вопросом дискутабельным и требует дальнейших исследований.

3. Smith O.P., Hann I.M., Chessells J.M. et al. Haematological abnormalities in Shwachman-Diamond syndrome. Br. J. Haematol. 1996; 94(2): 279-84. DOI: 10.1046/j.1365-2141.1996.d01-1788.x

4. Burroughs L., Woolfrey A., Shimamura A. Shwachman-Diamond syndrome: a review of the clinical presentation, molecular pathogenesis, diagnosis, and treatment. Hematol. Oncol. Clin. North Am. 2009; 23(2): 233-48. DOI 10.1016/j.hoc.2009.01.007 
5. Ипатова М.Г., Кучев С.И., Шумилов П.В. и др. Краткие клинические рекомендации по ведению больных с синдромом Швахмана Даймонда. Педиатрия. Журнал им. Г.Н. Сперанского. 2016; 95(6): 181-6. [Ipatova M.G., Kutsev S.I., Shumilov P.V. et al. Summary of clinical recommendations for the management of patients with Schwachman Diamond syndrome. Pediatria. Journal named after G.N. Speransky. 2016; 95(6): 181-6. (in Russian)]

6. Dror Y., Durie P., Ginzberg H. et al. Clonal evolution in marrows of patients with Shwachman-Diamond syndrome: a prospective 5-year follow-up study. Exp. Hematol. 2002, 30(7): 659-69. D0I: 10.1016/s0301-472x(02)00815-9

7. Babushok D.V., Bessler M., Olson T.S. Genetic predisposition to myelodysplastic syndrome and acute myeloid leukemia in children and young adults. Leuk. Lymphoma. 2016; 57(3): 520-36. DOI: 10.3109/10428194.2015.1115041

8. Goobie S., Popovic M., Morrison J. et al. Shwachman-Diamond syndrome with exocrine pancreatic dysfunction and bone marrow failure maps to the centromeric region of chromosome 7. Am. J. Hum. Genet. 2001; 68(4): 1048-54. DOI: 10.1086/319505

9. Minelli A., Nicolis E., Cannioto Z. et al. Incidence of Shwachman-Diamond syndrome in Italy. Pediatr. Blood Cancer. 2012; 59(7): 1334-5. D0I: 10.1002/ pbc. 24260

10. Alter B.P., Giri N., Savage S.A. et al. Cancer in the National Cancer Institute inherited bone marrow failure syndrome cohort after fifteen years of follow-up. Haematologica. 2018; 103(1): 30-9. DOI: 10.3324/haematol.2017.178111

11. Hashmi S.K., Allen C., Klaassen R. et al. Comparative analysis of ShwachmanDiamond syndrome to other inherited bone marrow failure syndromes and genotype-phenotype correlation. Clin. Genet. 2011; 79(5): 448-58. DOI: 10.1111/j.1399-0004.2010.01468.x

12. Mukhina A.A., Kuzmenko N.B., Rodina Y.A. et al. Primary immunodeficiencies in Russia: data from the National Registry. Front. Immunol. 2020; 11: 1491. DOI: 10.3389/fimmu.2020.01491

13. Woloszynek J.R., Rothbaum R.J., Rawls A.S. et al. Mutations of the SBDS gene are present in most patients with Shwachman-Diamond syndrome. Blood. 2004; 104(12): 3588-90. DOI: 10.1182/blood-2004-04-1516

14. Nicolis E., Bonizzato A., Assael B.M. et al. Identification of novel mutations in patients with Shwachman-Diamond syndrome. Hum. Mutat. 2005; 25(4): 410. DOI: 10.1002/humu.9324

15. Donadieu J., Fenneteau 0., Beaupain B. et al. Classification of and risk factors for hematologic complications in a French national cohort of 102 patients with Shwachman-Diamond syndrome. Haematologica. 2012; 97(9): 1312-9. DOI: 10.3324/haematol.2011.057489

16. Zhang S., Shi M., Hui C.C. et al. Loss of the mouse ortholog of the ShwachmanDiamond syndrome gene (Sbds) results in early embryonic lethality. Mol. Cell Biol. 2006; 26(17): 6656-63. DOI: 10.1128/MCB.00091-06

17. Nakashima E., Mabuchi A., Makita Y. et al. Novel SBDS mutations caused by gene conversion in Japanese patients with Shwachman-Diamond syndrome. Hum. Genet. 2004; 114(4): 345-8. D0I: 10.1007/s00439-004-1081-2

18. Steele L., Rommens J.M., Stockley T. et al. De novo mutations causing Shwachman-Diamond syndrome and a founder mutation in SBDS in the French Canadian population. J. Investig. Genomics. 2014; 1(2): 38-41. DOI: 10.15406/jig.2014.01.00008

19. Dror Y., Donadieu J., Koglmeier J. et al. Draft consensus guidelines for diagnosis and treatment of Shwachman-Diamond syndrome. Ann. NY Acad. Sci. 2011; 1242(1): 40-55. DOI: 10.1111/j.1749-6632.2011.06349.x

20. Tummala H., Walne A.J., Williams M. et al. Affiliations expand. DNAJC21 mutations link a cancer-prone bone marrow failure syndrome to corruption in $60 S$ ribosome subunit maturation. Am. J. Hum. Genet. 2016; 99(1): 115-24. DOI: 10.1016/j.ajhg.2016.05.002

21. Dhanraj S., Matveev A., Li H. et al. Biallelic mutations in DNAJC21 cause Shwachman-Diamond syndrome. Blood. 2017; 129(11): 1557-62. DOI: 10.1182/blood-2016-08-735431

22. Stepensky P., Chacón-Flores M., Kim K.H. et al. Mutations in EFL1, an SBDS partner, are associated with infantile pancytopenia, exocrine pancreatic insufficiency and skeletal anomalies in a Shwachman-Diamond like syndrome. J. Med. Gen. 2017; 54(8): 558-66. DOI: 10.1136/jmedgenet-2016-104366

23. Carapito R., Konantz M., Paillard C. et al. Mutations in signal recognition particle SRP54 cause syndromic neutropenia with Shwachman-Diamond-like features. J. Clin. Invest. 2017; 127(11): 4090-103. DOI: 10.1172/JCI92876

24. Bellanne-Chantelot C., Schmaltz-Panneau B., Marty C. et al. Mutations in the SRP54 gene cause severe congenital neutropenia as well as ShwachmanDiamond-like syndrome. Blood. 2018; 132(12): 1318-31. DOI: 10.1182/ blood-2017-12-820308
25. Tafforeau L., Zorbas C., Langhendries J.L. et al. The complexity of human ribosome biogenesis revealed by systematic nucleolar screening of PrerRNA processing factors. Mol. Cell. 2013; 51(4): 539-51. DOI: 10.1016/j. molcel.2013.08.011

26. de la Cruz J., Karbstein K., Woolford J.L. Jr. Functions of ribosomal proteins in assembly of eukaryotic ribosomes in vivo. Annu Rev. Biochem. 2015; 84 93-129. DOI: 10.1146/annurev-biochem-060614-033917

27. Klinge S., Voigts-Hoffmann F., Leibundgut M. et al. Atomic structures of the eukaryotic ribosome. Trends Biochem. Sci. 2012; 37(5): 189-98. DOI: 10.1016/j.tibs.2012.02.007

28. Austin K.M., Gupta M.L. Jr, Coats S.A. et al. Mitotic spindle destabilization and genomic instability in Shwachman-Diamond syndrome. J. Clin. Invest. 2008, 118(4): 1511-18. DOI: 10.1172/JCI33764

29. Finch A.J., Hilcenko C., Basse N. et al. Uncoupling of GTP hydrolysis from eIF6 release on the ribosome causes Shwachman-Diamond syndrome. Genes Dev. 2011; 25(9): 917-29. D0I: 10.1101/gad.623011

30. Gartmann M., Blau M., Armache J.P. et al. Mechanism of eIF6-mediated inhibition of ribosomal subunit joining. J. Biol. Chem. 2010; 285(20): 14848-51. DOI: 10.1074/jbc.C109.096057

31. Austin K.M., Leary R.J., Shimamura A. The Shwachman-Diamond SBDS protein localizes to the nucleolus. Blood. 2005; 106(4): 1253-8. DOI: 10.1182/ blood-2005-02-0807

32. Menne T.F., Goyenechea B., Sánchez-Puig N. et al. The Shwachman-BodianDiamond syndrome protein mediates translational activation of ribosomes in yeast. Nat. Genet. 2007; 39(4): 486-95. DOI: 10.1038/ng1994

33. Wong C.C., Traynor D., Basse N. et al. Defective ribosome assembly in Shwachman-Diamond syndrome. Blood. 2011; 118(16): 4305-12. DOI: 10.1182/blood-2011-06-353938

34. Keenan R.J., Freymann D.M., Stroud R.M. et al. The signal recognition particle. Annu Rev. Biochem. 2001; 70: 755-75. DOI: 10.1146/annurev. biochem.70.1.755

35. Akopian D., Shen K., Zhang X. et al. Signal recognition particle: an essential protein-targeting machine. Annu Rev. Biochem. 2013; 82: 693-721. DOI: 10.1146/annurev-biochem-072711-164732

36. Drygin D., O'Brien S.E., Hannan R.D. et al. Targeting the nucleolus for cancerspecific activation of p53. Drug Discov. Today. 2014; 19(3): 259-65. DOI: 10.1016/j.drudis.2013.08.012

37. Quin J.E., Devlin J.R., Cameron D. et al. Targeting the nucleolus for cancer intervention. Biochim. Biophys. Acta. 2014; 1842(6): 802-16. DOI: 10.1016/j.bbadis.2013.12.009

38. Ganapathi K.A., Austin K.M., Lee C.-S. et al. The human Shwachman-Diamond syndrome protein, SBDS, associates with ribosomal RNA. Blood. 2007; 110(5): 1458-65. DOI: 10.1182/blood-2007-02-075184

39. Draptchinskaia N., Gustavsson P., Andersson B. M. et al. The gene encoding ribosomal protein $\mathrm{S} 19$ is mutated in Diamond-Blackfan anaemia. Nat. Genet. 1999; 21(2): 169-75. DOI: 10.1038/5951

40. Ebert B.L. Deletion $5 q$ in myelodysplastic syndrome: a paradigm for the study of hemizygous deletions in cancer. Leukemia. 2009; 23(7): 1252-6. DOI: 10.1038/leu.2009.53

41. Heiss N.S., Knight S.W., Vulliamy T.J. et al. X-linked dyskeratosis congenita is caused by mutations in a highly conserved gene with putative nucleolar functions. Nat. Genet. 1998; 19(1): 32-8. DOI: 10.1038/ng0598-32

42. Ridanpää M., van Eenennaam H., Pelin K. et al. Mutations in the RNA component of RNase MRP cause a pleiotropic human disease, cartilage-hair hypoplasia. Cell. 2001; 104: 195-203. DOI: 10.1016/s0092-8674(01)00205-7

43. Vulliamy T.J., Marrone A., Knight S.W. et al. Mutations in dyskeratosis congenita: their impact on telomere length and the diversity of clinical presentation. Blood. 2006; 107(7): 2680-5. D0I: 10.1182/blood-2005-07-2622

44. Warren A.J. Molecular basis of the human ribosomopathy ShwachmanDiamond syndrome. Adv. Biol. Regul. 2018; 67: 109-27. D0I: 10.1016/j. jbior.2017.09.002

45. Gazda H.T., Zhong R., Long L. et al. RNA and protein evidence for haploinsufficiency in Diamond-Blackfan anaemia patients with RPS19 mutations. Br. J. Haematol. 2004; 127(1): 105-13. D0I: 10.1111/j.1365-2141.2004.05152.x

46. Mason P.J., Wilson D.B., Bessler M. Dyskeratosis congenital - a disease of dysfunctional telomere maintenance. Curr. Mol. Med. 2005; 5(2): 159-70. DOI: $10.2174 / 1566524053586581$

47. Vlachos A., Klein G.W., Lipton J.M. The Diamond-Blackfan Anemia Registry: tool for investigating the epidemiology and biology of Diamond-Blackfan anemia. J. Pediatr. Hematol. Oncol. 2001; 23(6): 377-82. DOI: 10.1097/00043426200108000-00015 D 